\title{
Singularities in cylindrical vector beams
}

\author{
Manish Verma, Sushanta Kumar Pal, Stuti Joshi, P. Senthilkumaran, Joby \\ Joseph \& H.C. Kandpal
}

To cite this article: Manish Verma, Sushanta Kumar Pal, Stuti Joshi, P. Senthilkumaran, Joby Joseph \& H.C. Kandpal (2015) Singularities in cylindrical vector beams, Journal of Modern Optics, 62:13, 1068-1075, DOI: 10.1080/09500340.2015.1019384

To link to this article: http://dx.doi.org/10.1080/09500340.2015.1019384

\section{曲 Published online: 11 Mar 2015.}

\section{Submit your article to this journal}

Џلll Article views: 125

Q View related articles $\sqsubset$

View Crossmark data $₫$ 


\title{
Singularities in cylindrical vector beams
}

\author{
Manish Verma ${ }^{\mathrm{a}, \mathrm{b} *}$, Sushanta Kumar Pal ${ }^{\mathrm{b}}$, Stuti Joshi ${ }^{\mathrm{a}}$, P. Senthilkumaran ${ }^{\mathrm{b}}$, Joby Joseph ${ }^{\mathrm{b}}$ and H.C. Kandpal ${ }^{\mathrm{a}}$ \\ ${ }^{a}$ Quantum Optics and Photon Physics, CSIR - National Physical Laboratory, New Delhi, India; ${ }^{b}$ Department of Physics, Indian \\ Institute of Technology Delhi, New Delhi, India
}

(Received 29 December 2014; accepted 10 February 2015)

\begin{abstract}
Cylindrical vector beams with azimuthal and radial polarization distributions are studied for singularities. It is shown experimentally that these beams have screw dislocation as well as edge dislocation at the same time. The relation between phase and polarization of light beam is the key to understand this fact. We envisage that this has potential application in phase synthesis using polarization engineering. Further, the polarization singularities in these inhomogeneously polarized beams are examined by measuring Stokes parameters across the cross-section of these beams.
\end{abstract}

Keywords: polarization; singular optics; interference

\section{Introduction}

Optical vortex, also called screw dislocation [1] or phase singularity, is characterized by intensity null within the cross-section of a beam. At the intensity null point, the phase is undefined and in its neighborhood, the wavefront is helical in shape $[2,3]$. The topology is such that all the wavefronts which are disconnected and separated by distance $\lambda$ with each other in an otherwise non-singular beam, become connected thereby forming a single wavefront filling the entire space. The optical vortices can be characterized by various interferometry techniques [4-7]. In two beam interferometry, fork-type fringes (characteristic of phase singularity) appear at the singular region. Optical vortices have significant importance and applications in classical and quantum optics [8-13]. This type of singularity is scalar in nature. There is another type of singularity called vector singularity, which is present whenever polarization or some of the parameters (Stokes parameter, degree of polarization) used to define the state of polarization become indeterminate [14-16]. In recent years, the beams having spatially inhomogeneous polarization distribution across its cross-section, known as cylindrical vector (CV) beams, received a lot of attention in the scientific community. Electric fields in the focal region of such beams have unique properties [15-19]. Radially polarized (RP) beams and azimuthally polarized (AP) beams are two types of $\mathrm{CV}$ beams having potential applications in many areas [20-27].

In this paper, we have studied RP and AP beams generated using liquid crystal-based polarization converter (PC). These beams have inhomogeneous polarization distribution across its cross-section and an intensity null at the centre. Decomposing the RP/AP beams into its orthogonal polarization states can reveal the presence of point or line phase defects in the beam. By interfering RP/AP beams with a circularly polarized beam, fork-type fringe appear at the center which shows the presence of a phase singularity of unit charge at the center of the beam. Similarly, by interfering RP/AP beams with linearly polarized beam, fringes shift along the phase discontinuity line showing the presence of edge dislocation in the $\mathrm{CV}$ beams. Further, the polarization singularities present across the beam cross-section are characterized by measuring the Stokes parameters over its cross-section.

\section{Phase defects}

Decomposition of a polarization state into its orthogonal components $[28,29]$ holds the key in extracting the beam with line or point phase defects from a CV beam. There is an infinite number of ways that the decomposition can be thought of. For any state of polarization defined by a point on the Poincare sphere, its orthogonal state can be located at a diametrically opposite point on the sphere. Therefore, any state can be decomposed into two orthogonal states with a phase difference between them. The decomposition of RP/AP beam into its orthogonal circular polarization components shows that the decomposed beams are singular, each with a point phase defect. If the decomposition is such that the two decomposed beams are linearly orthogonally polarized, the phase front of the decomposed beams possess line phase defect. In fact, the number of ways decomposition of inhomogeneously polarized beams can be made, offers possibilities of realizing wavefronts of different shapes from an appropriately polarization-engineered single beam.

\footnotetext{
*Corresponding author. Email: mv4manishverma@gmail.com 
The RP and AP light can be described as a sum of left circularly polarized (LCP) and right circularly polarized (RCP) light with opposite helical phases [28],

$$
\begin{aligned}
& \vec{E}_{R P / A P}(x, y) \\
& \quad=E_{0}(\hat{x}+i \hat{y}) \exp \left[i\left(k z-\omega t+m \phi(x, y)+\phi_{0}\right)\right] \\
& \quad+E_{0}(\hat{x}-i \hat{y}) \exp \left[i\left(k z-\omega t-m \phi(x, y)-\phi_{0}\right)\right]
\end{aligned}
$$

where $\phi(x, y)=\tan ^{-1}(y / x)$ gives the azimuthal phase variation, $\pm m$ is the topological charge of the singular beams, $\phi_{0}=0$ for a RP beam and $\phi_{0}=\pi / 2$ for an AP beam. Since $\phi(x, y)$ is not constant, the propagation vector $\vec{k}$ has transverse $(x, y)$ components arising due to $\nabla \phi(x, y)$ and are ignored here. Moreover, the superposition of positive and negative vortices produce circulating $\nabla \phi(x, y)$ transverse components that cancels out. These transverse components of $\vec{k}$ are responsible for internal energy flows within the transverse plane [30-33]. The electric field vector of a tilted interfering RCP/LCP beam, having propagation vector $\vec{k}$ making a small angle $\alpha$ with $z$-axis in the $x-z$ plane, is given by:

$$
\begin{aligned}
& \vec{E}_{R C P / L C P}(x, y) \\
& \quad=E_{0}\{(\hat{x} \pm i \hat{y}) \exp [i(k z \cos \alpha+k x \sin \alpha-\omega t)]\}
\end{aligned}
$$

Since $\alpha$ is small, the longitudinal component of $\vec{E}_{R C P / L C P}$ along the $z$-direction is ignored. The interference pattern is given by:

$$
\begin{aligned}
I(x, y)= & \left|E_{R P / A P}(x, y)\right|^{2}+\left|E_{R C P / L C P}(x, y)\right|^{2} \\
& +2\left|E_{R P / A P}(x, y) \times E_{R C P / L C P}^{*}(x, y)\right|
\end{aligned}
$$

The interference pattern as described by Equation (3), observed at any $z=z_{1}$ plane where $z_{1}$ a constant, contains fork fringes that indicate the presence of phase singularity in the RP and AP beams. Note that the orthogonal polarization components do not interfere. For example, the RCP component of RP/AP beam interferes with the interfering RCP beam to yield fork fringes due to the presence of the helical phase term $m \phi$ in Equation (1). The LCP component of RP/AP merely adds to the background. In Equation (3), the formation of fringes (constructive interference) is governed by the term

$$
\cos \left(k x \sin \alpha-\left(m \phi+\phi_{0}\right)\right)=+1
$$

where the period of the fringes (away from the vortex core) is decided by $\alpha$ and the number of extra fringes in the fork formation is decided by $m$.

To observe line phase defect, the RP beam can be decomposed into linearly polarized beams. In Figure 1, we tried to depict how this decomposition can be done, where the presence of phase step is illustrated in red color. For radial polarization, the decomposition is such that the direction of linear polarization, in the two halves of each of the beams, is perpendicular to the phase step, whereas the direction of linear polarization is oriented parallel to the phase step for the case of AP beam decomposition. The electric field vector of the RP plane wave is given by:

$$
\begin{aligned}
\vec{E}_{R P}(x, y)= & E_{1}(x, y) \hat{x} \operatorname{sgn}[x] \exp [i(k z-\omega t)] \\
& +E_{2}(x, y) \hat{y} \operatorname{sgn}[y] \exp [i(k z-\omega t)]
\end{aligned}
$$

where $\operatorname{sgn}[x]$ and $\operatorname{sgn}[y]$ are signum functions defined as:

$$
\operatorname{sgn}[x]= \begin{cases}+1 & \text { for } x \geq 0 \\ -1 & \text { for } x<0\end{cases}
$$

When the RP beam given by Equation (5), interferes with a linearly polarized plane wave whose electric field vector is given by:

$$
\vec{E}_{L P}(x, y)=E_{0} \hat{y} \exp [i(k z \cos \alpha+k x \sin \alpha-\omega t)]
$$

the interference fringes can be observed at $x y$-plane. The $y$-component of the radial polarization interferes with the $y$-component of the reference plane wave, while the $x$-component of the radial polarization adds to the background intensity in the interference pattern. The straight line fringes running parallel to $y$-axis will have a step discontinuity where the line phase defect is present. This edge dislocation is along $x=0$ line in the $x y$-plane.

Similarly, azimuthal polarization can be thought of superposition of two orthogonal linear polarizations with the presence of signum function in its phase distributions.

$$
\begin{aligned}
\vec{E}_{A P}(x, y)= & E_{3}(x, y) \hat{y} \operatorname{sgn}[x] \exp [i(k z-\omega t)] \\
& +E_{4}(x, y) \hat{x} \operatorname{sgn}[y] \exp [i(k z-\omega t)]
\end{aligned}
$$

The interference of AP beam with a linearly polarized plane wave will reveal the presence of phase step by way of fringe shift. In Equations (5) and (8), the amplitudes $E_{i}(x, y)$ are not constants and are functions of $x$ and $y$. For example, $E_{1}(x, y)=\left|E_{0} \cos \beta(x, y)\right|$ and $\beta$ is measured with respect to the line perpendicular to the phase dislocation (jump). Basically, we have written the bipolar nature of $\cos \beta$ variation as a product of a unipolar function (always positive) and a signum function. The amplitudes $E_{i}(x, y)$ can be thought (only the amplitude part) of the lobes of a $\mathrm{HG}_{10}$ or $\mathrm{HG}_{01}$ mode in the two halves of the beam. Note in the $\mathrm{HG}_{10 / 01}$ modes, the SOPs in the two lobes are same but with a $\pi$ phase difference.

\section{Polarization singularities}

To observe the presence of polarization singularities in the azimuth and in the ellipticity, the Stokes parameters are experimentally observed and analyzed. The polarization 

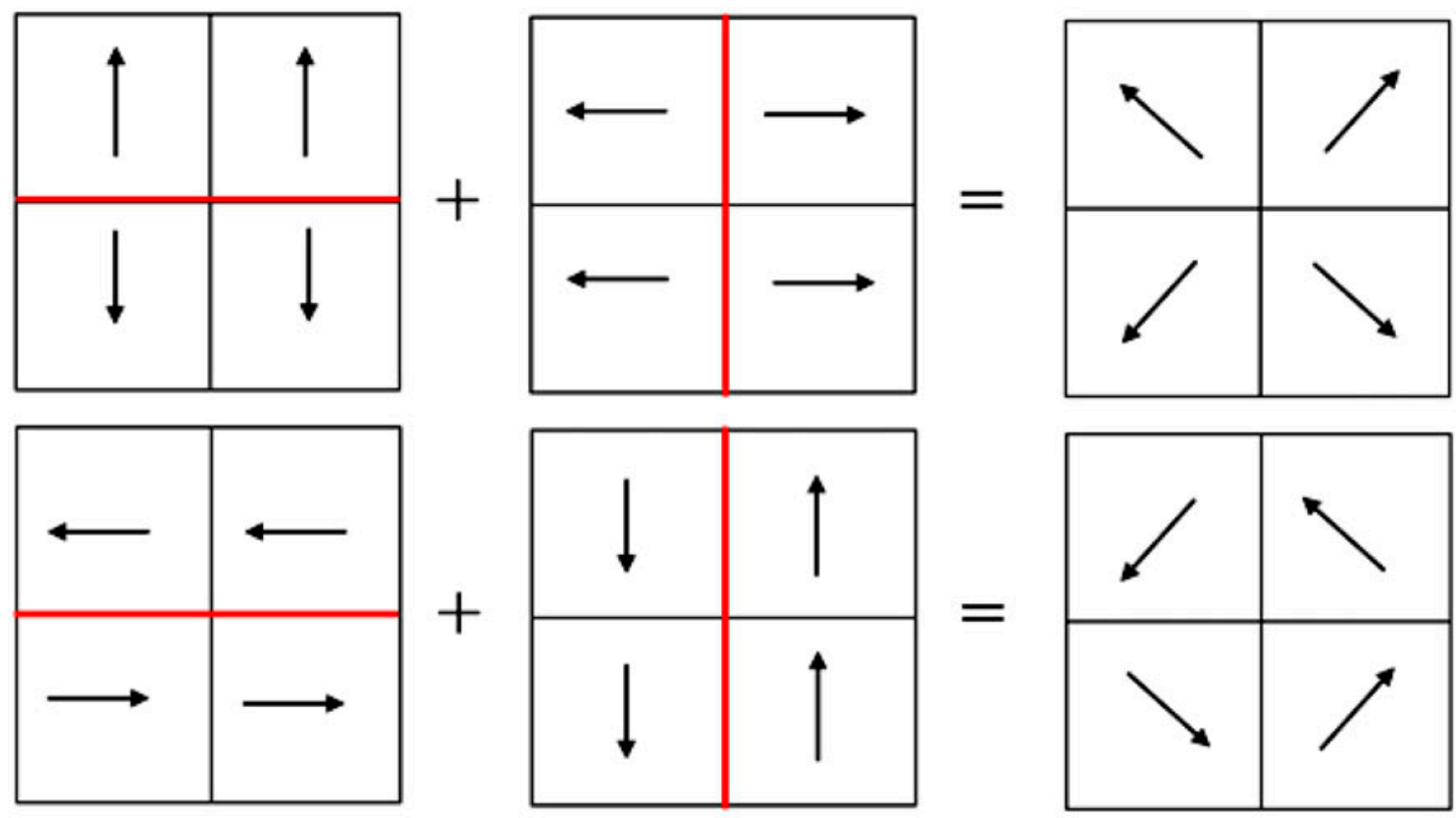

Figure 1. Schematic to understand the presence of phase steps in the RP and AP beams. (The colour version of this figure is included in the online version of the journal.)

state of a vector beam can be represented completely by the Poincare sphere [34]. In general, an elliptically polarized beam with its major axis making an angle $\psi$ with the $\mathrm{X}$-axis (horizontal axis) is represented by a point $\mathrm{P}$ on the surface of the Poincare sphere (as shown in Figure 2) of radius $\mathrm{S}_{0}$. The co-ordinate axes $\mathrm{S}_{1}, \mathrm{~S}_{2}, \mathrm{~S}_{3}$, and $\mathrm{S}_{0}$ are the Stokes parameters which are experimentally calculated using a quarter wave plate followed by a linear polarizer. The angle $\psi$ determines the orientation of the major axis of the ellipse of the elliptically polarized light and the angle $2 \chi$ determines the ellipticity of the polarization state. If the latter is zero, the polarization state is linear. On the other hand, if it is $\pm \pi / 2$, the polarization is circular (either right handed or left handed). If $I(\phi, \theta)$ represents the

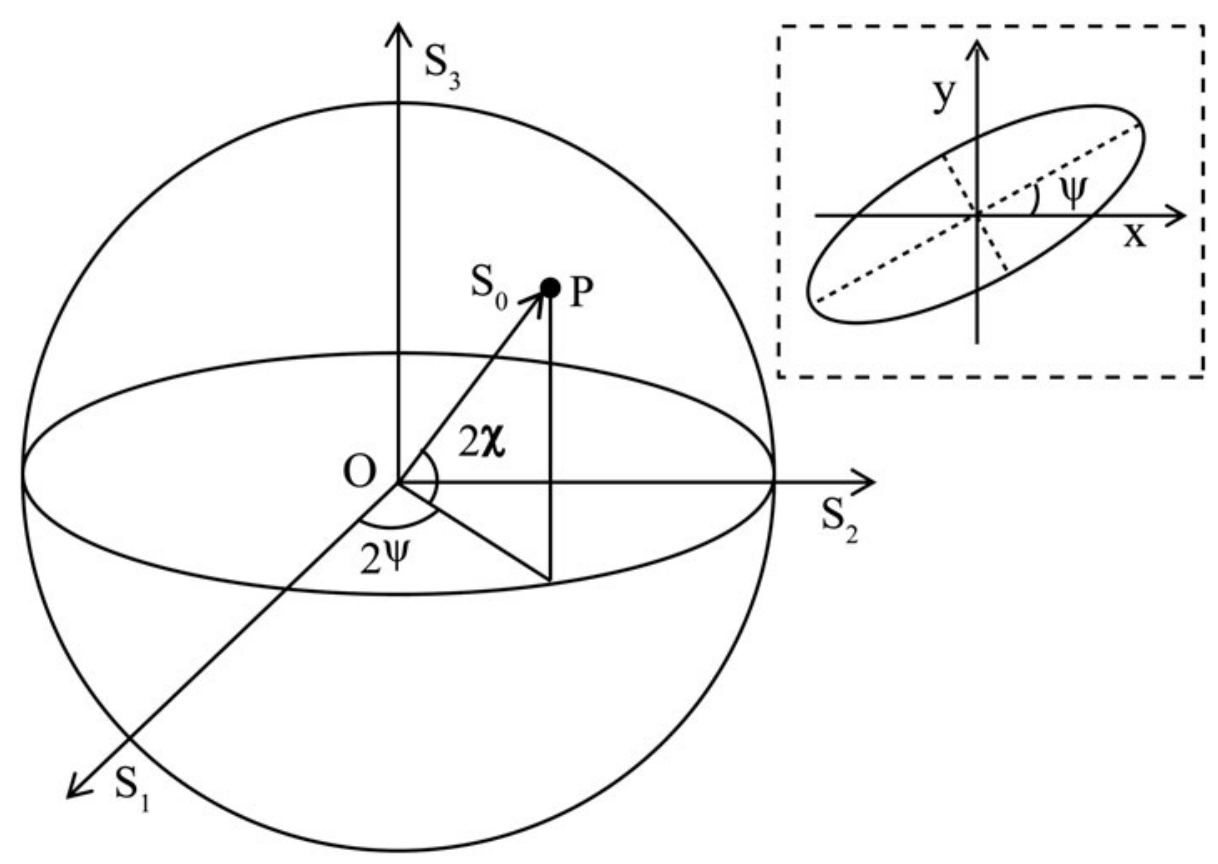

Figure 2. Elliptically polarized light represented by the polarization ellipse and the Poincare sphere. 


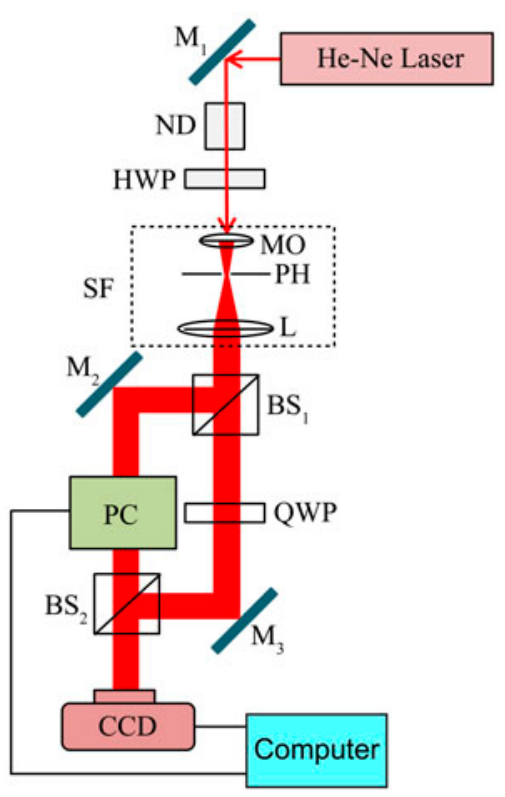

Figure 3. Setup for observing the interference pattern of RP/ AP beam with a circularly polarized beam. M, ND, HWP, SF, $\mathrm{PC}, \mathrm{QWP}, \mathrm{MO}, \mathrm{PH}, \mathrm{L}$, and $\mathrm{BS}$ refers to mirror, variable neutral density filter, half-wave plate, spatial filter, polarization converter, quarter wave plate, microscopic objective, pin hole, converging lens, and non-polarizing 50-50 beam splitter, respectively, where $L=15 \mathrm{~cm}, \mathrm{PH}=25 \mu \mathrm{m}, \mathrm{MO}=10 \times$. (The colour version of this figure is included in the online version of the journal.)

intensity recorded at a CCD camera when the fast axis of the quarter wave plate makes an angle $\phi$ and the polarizer axis makes an angle $\theta$ with $x$-axis, the four Stokes parameters are given by [35]:

$$
\begin{gathered}
S_{0}=I(0,0)+I(0,90) \\
S_{1}=I(0,0)-I(0,90) \\
S_{2}=I(45,45)-I(45,135) \\
S_{3}=I(0,45)-I(0,135)
\end{gathered} .
$$
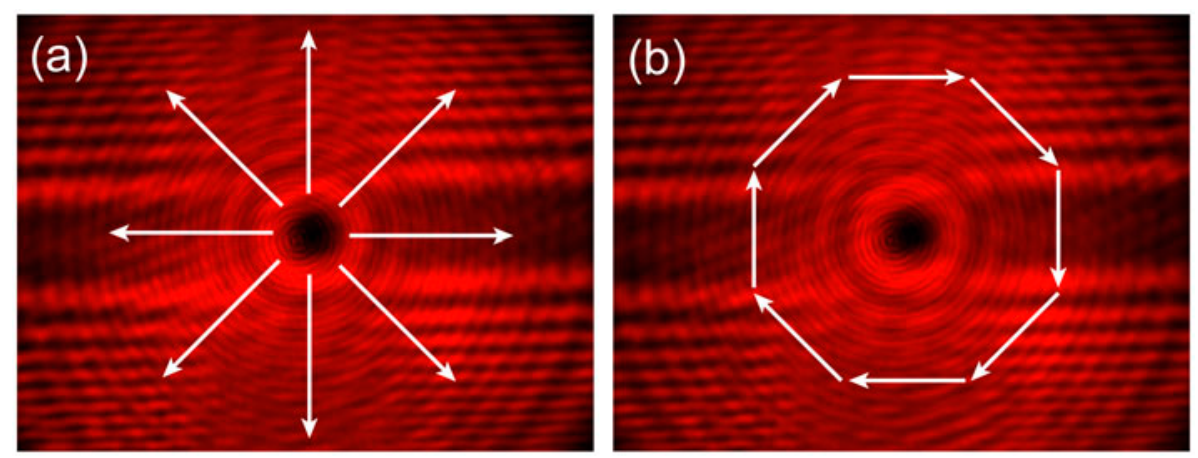

The angles $\psi$ and $\chi$ can be easily calculated using Equation (9), where

$$
\begin{aligned}
& \psi=\frac{1}{2} \tan ^{-1}\left(S_{2} / S_{1}\right) \\
& \chi=\frac{1}{2} \operatorname{Sin}^{-1}\left(S_{3} / S_{0}\right)
\end{aligned}
$$

At the center of the AP and RP beams, the presence of phase singularity ensures that $S_{0}=0$ with all other Stokes parameters $S_{1}=S_{2}=S_{3}=0$. Hence, the presence of polarization singularities can be seen by plotting the spatial distribution of $\psi$ and $\chi$. For both RP and AP beams, the handedness is not defined throughout the beam cross-section, since they are basically made up of linear polarization states.

\section{Experimental setup}

A 632.8-nm linearly polarized and intensity-stabilized $\mathrm{He}-\mathrm{Ne}$ laser (MellusGriot) beam is spatially filtered and expanded as shown in Figure 3. The beam is attenuated by a continuously variable neutral density filter ND and its polarization is aligned vertically by a half-wave plate HWP. The laser beam is then divided into two parts by a 50-50 beam splitter $\mathrm{BS}_{1}$. One part (vertically polarized) is fed to the PC through which the RP/AP beam is created (cross-sections of the generated RP and AP beams are shown in Figure 4). The other part is first made circularly polarized by insertion of a quarter-wave plate (QWP) and then combined with the RP/AP beam through another $50-50$ beam splitter $\mathrm{BS}_{2}$. The interference patterns for both RP and AP beams with RCP and LCP beams are captured by CCD camera and are shown in Figure $5(a)-(d)$, respectively. The simulated interference patterns for the same are shown in Figure 6(a)-(d). The RP/AP beam generated by the PC can be described from Equation (1), with a phase singularity of unit charge at the centre of the beam.

For line phase defect, in the experimental setup shown in Figure 3, the circularly polarized reference

Figure 4. (a) and $(b)$ show the cross-sections of RP and AP light beams generated from the polarization converter PC, respectively. Arrows shows the distribution of polarization across the beam cross-sections. (The colour version of this figure is included in the online version of the journal.) 

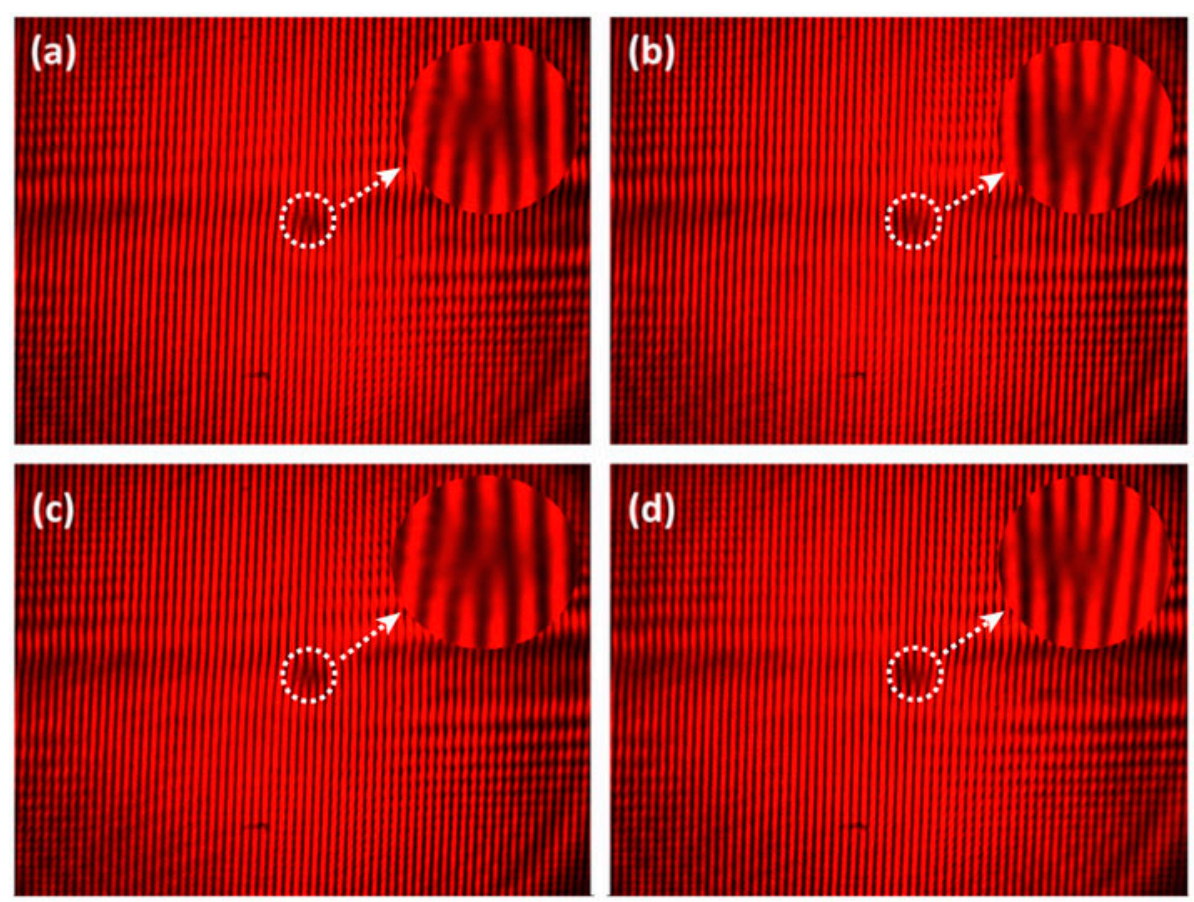

Figure 5. Interference pattern of (a) RP beam with LCP beam, $(b)$ RP beam with RCP beam, $(c)$ AP beam with LCP beam, and $(d)$ AP beam with RCP beam. The flipping of fork fringes can be seen by changing the handedness of the circularly polarized light. (The colour version of this figure is included in the online version of the journal.)

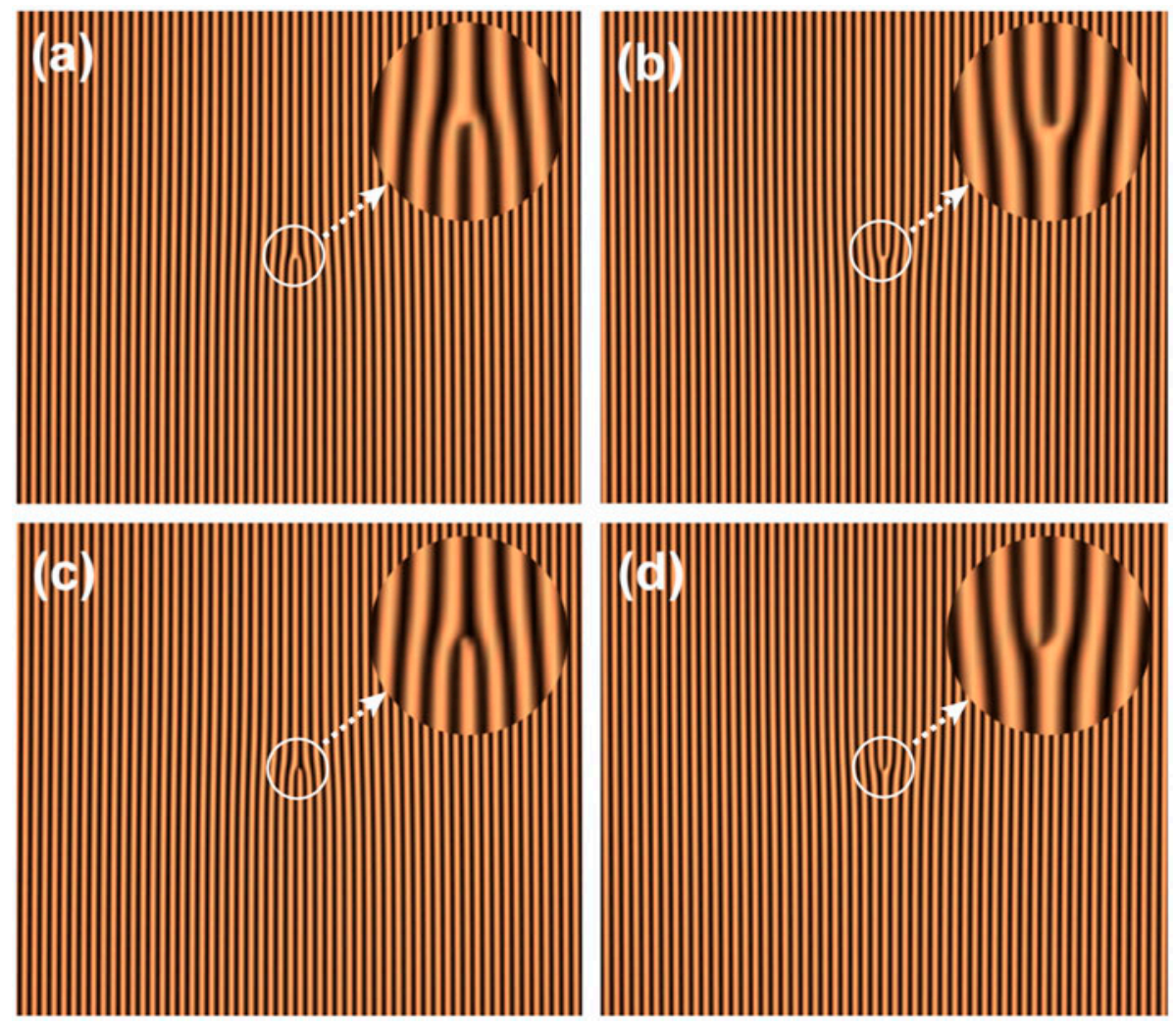

Figure 6. Simulated Interference pattern of $(a)$ RP beam with LCP beam, $(b)$ RP beam with RCP beam, $(c)$ AP beam with LCP beam, and $(d)$ AP beam with RCP beam. (The colour version of this figure is included in the online version of the journal.) 

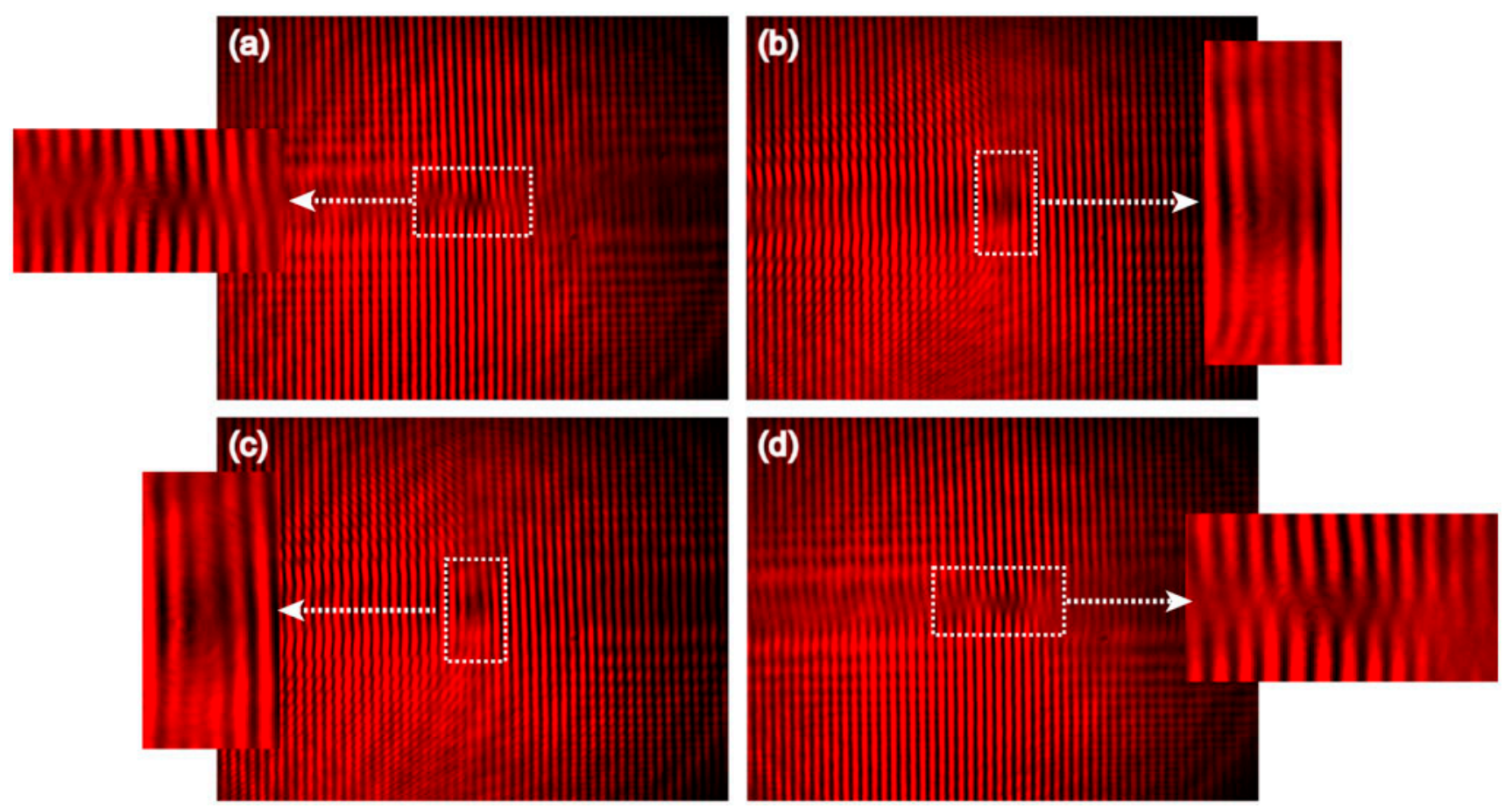

Figure 7. (a) and (b) Interference pattern of RP beam with linearly polarized light having polarization direction along vertical and horizontal direction, respectively. $(c)$ and $(d)$ Interference pattern of AP beam with linearly polarized light having polarization direction along vertical and horizontal direction, respectively. (The colour version of this figure is included in the online version of the journal.)

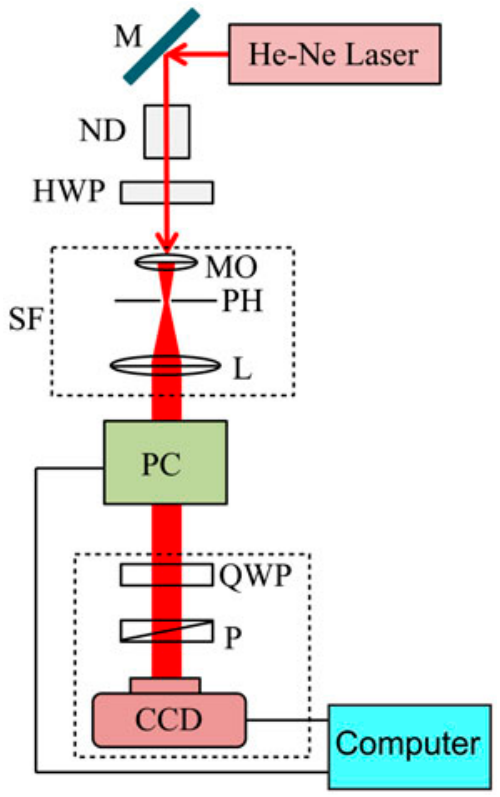

Figure 8. Experimental setup for determination of the Stokes parameters. QWP and $\mathrm{P}$ refer to quarter-wave plate and analyzer, respectively. (The colour version of this figure is included in the online version of the journal.)

beam is replaced by a plane polarized beam and is made to interfere the RP/AP beam. The observed interference patterns are shown in Figure $7(a)-(d)$. Fringes in the field of view are shifted by half the period (Figure 7(a) and $(d)$ ) where the phase step is present and this indicates the presence of the edge dislocation in the RP/AP beam. Since the tilt of the interfering beam is kept same, the phase steps in Figure $7(b)$ and $(c)$, are not seen clearly and it can be seen if we change the tilt of fringe orientation.

The setup for calculating the Stokes parameters of RP/AP beam is shown in Figure 8. The Stokes parameters are calculated with the aid of a quarter-wave plate followed by a linear polarizer and using Equation (9). The distribution of the angles $\psi$ and $\chi$ over the crosssections of both RP and AP beams is calculated from Equation (10) and shown in Figure 9. In Figure 9(a) and $(c)$, the distribution of $\psi$ shows the presence of vortex at the centre. This indicates that at the center $\psi$ is undefined in both RP and AP. In their immediate neighborhood, rapid changes in the orientation of the major axis occur which is typical of vortex structure. Since $S_{3}$ is zero for both the RP and AP beams the ellipticity is zero all across the beam as shown in Figure $9(b)$ and $(d)$.

\section{Results and discussion}

The presence of phase singularity at the center of the beam is observed from the interference of the RP/AP beam with a RCP/LCP beam shown in Figure 5. In the interference pattern, a fork fringe appears at the center 

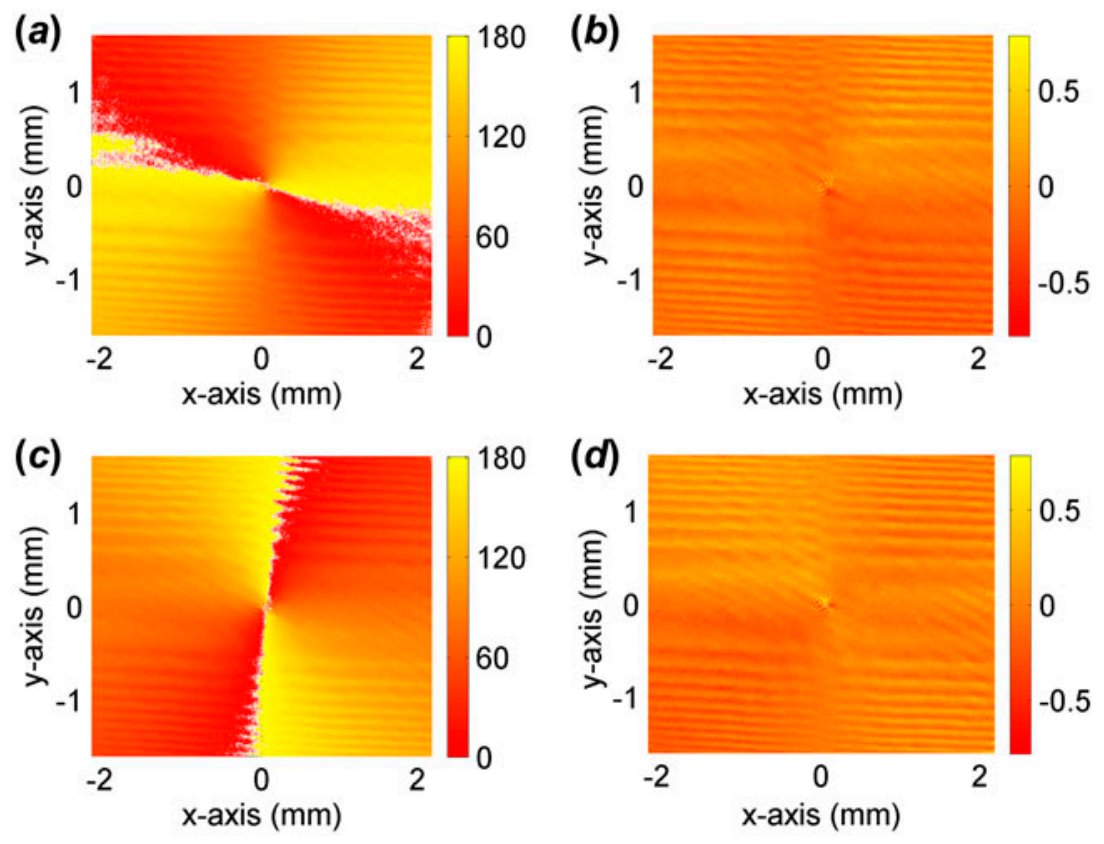

Figure 9. (a) and (b) distribution of the angles $\psi$ and $\chi$ over the cross-section of the RP light beam respectively, $(c)$ and $(d)$ distribution of the angles $\psi$ and $\chi$ over the cross-section of the AP light beam, respectively. Color bars show the angles in degrees. (The colour version of this figure is included in the online version of the journal.)

which indicates the presence of phase singularity of unit charge at the centre of the RP/AP beam. The decomposition of RP/AP into its linear polarization components reveals a line phase defect instead of point phase defect. This can be seen by the fringe shift between the two halves of the fringe pattern shown in Figure 7. It is very interesting to note that depending upon the way the decomposition of a polarization state into its orthogonal components, the wavefront with different characteristics can be seen. There is a polarization singularity present at the center of the beam because the polarization is undefined around that region. To determine the nature of the polarization singularity, the Stokes parameters for both RP and AP beams are measured using Equation (9). The angles $\psi$ and $\chi$ are calculated from the measured Stokes parameters from Equation (10). The distributions for the said angles across the cross-section for both RP and AP light beams are shown in Figure 9. Some fringes appeared in the calculated values of the angles $\psi$ and $\chi$ which are due to low magnitude (low intensity) of the parameter $\mathrm{S}_{0}$ in those regions. The topological charge of the polarization singularity and the state of polarization, within the cross-section of the beam, is observed from the distribution of the angles $\psi$ and $\chi$. Since there is a total rotation of $2 \pi$ of the angle $\psi$ around the center of the beam for both radially and AP beams, the topological charge of the polarization singularity is of magnitude one. Similarly, the magnitude of the angle $\chi$ in the crosssection of the beams is almost zero which indicates that the polarization state of the beams is linear. Therefore, in this work, we have investigated the polarization properties of the radially and AP beams, as well as have characterized the presence of phase singularity at the center of the beam.

\section{Conclusion}

In conclusion, we have studied the RP and AP beams generated from a liquid crystal-based polarization converter. The generated beams have a ring-like intensity profile with an intensity minimum at its center. Interference of the RP and AP beams with a circularly polarized beam results in formations of fork-type fringes at the center of the interference pattern showing the presence of phase singularity of unit charge at the centre of the beam. The presence of edge dislocation in the RP and AP beam can be revealed using a linearly polarized beam as the reference beam in the interference setup. It is very interesting to note that depending upon the way the decomposition of a polarization state into its orthogonal components, the wavefront with different characteristics can be seen. In addition, the polarization properties of the inhomogeneously polarized beams are experimentally studied by measuring the Stokes parameters across the cross-section of both RP and AP beams. The distribution of the angles $\psi$ and $\chi$ across the cross-section of the RP and AP beams shows the presence of polarization singularity at their center. Therefore, in this work, the 
phase singularities as well as polarization singularities across the cross-section of the RP and AP beams are studied in detail. This study has considerable importance in the field of singular optics and wavefront shaping.

\section{Acknowledgements}

One of the authors, Manish Verma thanks the Council of Scientific and Industrial Research (CSIR) for giving financial support as a senior research fellowship. He also thanks the Director, CSIR-NPL and the Director, IIT-Delhi for giving permission to publish this paper and his colleagues Manoj and Brijesh for useful discussion.

\section{References}

[1] Nye, J.; Berry, M. Proc. Royal Soc. A: Math. Phys. Eng. Sci. 1974, 336, 165-190.

[2] Bazhenov, V.Y.; Soskin, M.; Vasnetsov, M. J. Mod. Opt. 1992, 39, 985-990.

[3] Padgett, M.; Allen, L. Contemp. Phys. 2000, 41, 275-285.

[4] Ghai, D.P.; Senthilkumaran, P.; Sirohi, R.S. Opt. Commun. 2008, 281, 1315-1322.

[5] Senthilkumaran P.; Masajada, J.; Sato, S., Int. J. Opt. 2012, 1-18, Article ID 517591.

[6] Moreno, I.; Davis, J.A.; Ruiz, I.; Cottrell, D.M. Opt. Express 2010, 18, 7173-7183.

[7] Vaity, P.; Banerji, J.; Singh, R.P. Phys. Lett. A 2013, 377, 1154-1156.

[8] Senthilkumaran, P. Appl. Opt. 2003, 42, 6314-6320.

[9] Padgett, M.; Allen, L. Opt. Quantum Electron 1999, 31, 1-12.

[10] Grier, D.G. Nature 2003, 424, 810-816.

[11] Gahagan, K.; Swartzlander, G., Jr. Opt. Lett. 1996, 21, 827-829.

[12] Dholakia, K.; Simpson, N.; Padgett, M.; Allen, L. Phys. Rev. A: Atom. Mol. Opt. Phys. 1996, 54, R3742.

[13] Mair, A.; Vaziri, A.; Weihs, G.; Zeilinger, A. Nature 2001, 412, 313-316.
[14] Jayasurya, Y.; Krishna Inavalli, V.; Viswanathan, N.K. Appl. Opt. 2011, 50, E131-E137.

[15] Oron, R.; Blit, S.; Davidson, N.; Friesem, A.A.; Bomzon, Z.; Hasman, E. Appl. Phys. Lett. 2000, 77, 3322-3324.

[16] Nesterov, A.; Niziev, V. J. Phys. D: Appl. Phys. 2000, 33, 1817.

[17] Ramachandran, S.; Kristensen, P.; Yan, M.F. Opt. Lett. 2009, 34, 2525-2527.

[18] Zhan, Q.; Leger, J.R. Opt. Exp. 2002, 10, 324-331.

[19] Youngworth, K.; Brown, T. Opt. Express 2000, 7, 77-87.

[20] Novotny, L.; Beversluis, M.; Youngworth, K.; Brown, T. Phys. Rev. Lett. 2001, 86, 5251.

[21] Zhan, Q. Opt. Lett. 2006, 31, 1726-1728.

[22] Zhan, Q. Opt. Express 2004, 12, 3377-3382.

[23] Dorn, R.; Quabis, S.; Leuchs, G. Phys. Rev. Lett. 2003, 91, 233901.

[24] Grosjean, T.; Courjon, D.; Spajer, M. Opt. Commun. 2002, 203, 1-5.

[25] Bashkansky, M.; Park, D.; Fatemi, F.K. Opt. Express 2010, 18, 212-217.

[26] Stalder, M.; Schadt, M. Opt. Lett. 1996, 21, 1948-1950.

[27] Maurer, C.; Jesacher, A.; Fürhapter, S.; Bernet, S.; RitschMarte, M. New J. Phys. 2007, 9, 78.

[28] Pancharatnam, S. Proc. Indian Acad. Sci. Sect. A 1956, 44, 247-262.

[29] Senthilkumaran, P. Appl. Opt. 1999, 38, 1311-1316.

[30] Bahl, M.; Senthilkumaran, P. J. Opt. Soc. Am. A 2012, 29, 2421-2427.

[31] Bekshaev, A.; Bliokh, K.Y.; Soskin, M. J. Opt. 2011, 13, 32053001 .

[32] Bekshaev, A.Y.; Soskin, M.S. Opt. Commun. 2007, 271, 332-348.

[33] Bahl, M.; Senthilkumaran, P. J. Opt. Soc. Am. A 2014, 31, 2046-2054.

[34] Born, M.; Wolf, E. Principles of Optics; Cambridge University Press: Cambridge, 1999.

[35] Mandel, L., Wolf E.; Optical Coherence and Quantum Optics; Cambridge University Press: Cambridge, 1995. 